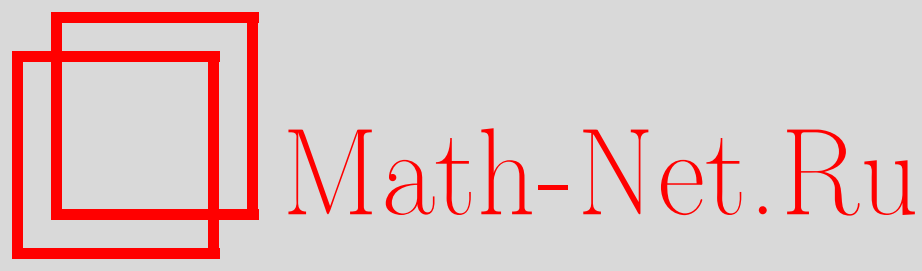

П. М. Ахметьев, О. В. Кунаковская, В. А. Кутвицкий, Замечание о диссипации интеграла магнитной спиральности, ТМФ, 2009, том 158, номер 1, 150-160

DOI: https://doi.org/10.4213/tmf6305

Использование Общероссийского математического портала Math-Net.Ru подразумевает, что вы прочитали и согласны с пользовательским соглашением http://www . mathnet.ru/rus/agreement

Параметры загрузки:

IP: 3.89 .185 .249

26 апреля 2023 г., $07: 25: 50$

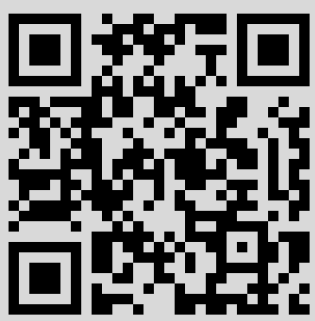




\title{
ЗАМЕЧАНИЕ О ДИССИПАЦИИ ИНТЕГРАЛА МАГНИТНОЙ СПИРАЛЬНОСТИ
}

\begin{abstract}
Доказано, что интеграл токовой спиральности для тонкой магнитной трубки прямо пропорционален величине скрученности Тw силовых линий, которая входит как слагаемое в формулу Калугариану. На этой основе анализируется закон изменения магнитной спиральности в проводящей среде с ненулевым коэффициентом магнитной диффузии, а также для уравнения фазового перехода магнитного поля в условиях ранней Вселенной.
\end{abstract}

Ключевые слова: магнитная гидродинамика, магнитная спиральность, диссипативные эффекты, формула Калугариану, формула Фуллера, Стандартная модель.

\section{1. ВВЕДЕНИЕ}

Вопрос о роли магнитной спиральности в эволюции магнитных полей в космосе является одним из центральных в современной магнитной гидродинамике космической среды. При его решении возникают разнообразные трудности: достаточно сказать, что плотность магнитной спиральности является нелокальной величиной (в нее входит векторный потенциал), а опыт работы с подобными законами сохранения в современной астрофизике практически отсутствует. В современную магнитную гидродинамику спиральность введена Моффатом и подробно рассматривалась в его монографии [1].

В настоящей работе предполагается, что пространство подразделено на конечное семейство попарно непересекающихся полноториев, называемых магнитными трубками, внутри которых сосредоточен носитель магнитного поля, и область, внешнюю по отношению к этим трубкам. Понятие магнитной трубки хорошо известно, оно играет первостепенную роль при изучении магнитных полей [2]. Для удобства читателя мы определяем это понятие в разделе 2.

* Институт земного магнетизма, ионосферы и распространения радиоволн им. Н. В. Пушкова РАН, г. Троицк, Московская обл., Россия.

E-mail: pmakhmet@mi.ras.ru, vak144@yandex.ru

${ }^{\dagger}$ Воронежский государственный университет, Воронеж, Россия. E-mail: ovk@math.vsu.ru 
Безусловно, важное значение имеет задача анализа магнитного поля в случае, если силовые линии поля не структурируются в трубки, а формируют более сложную трехмерную среду. Эта проблема многоаспектна, непроста и в настоящей работе не рассматривается. Впервые она была рассмотрена в работе [3] (см. также учебник [4]).

Магнитная спиральность для системы трубок прежде всего требует изучения внутренних (локальных) характеристик поля в каждой трубке: поток $\Phi$ поля через трансверсальное сечение трубки и скрученность Тw поля относительно центральной линии трубки. Кроме того, требуется изучить пространственное расположение каждой трубки (ее заузленность и, в частности, величину Wr перекручивания центральной линии), а также взаимное расположение системы трубок в пространстве. Простейшей числовой характеристикой их взаимного расположения служит коэффициент зацепления центральных линий $C, C^{\prime}$ выбранной пары магнитных трубок, который вычисляется с помощью интеграла Гаусса

$$
\operatorname{lk}\left(C, C^{\prime}\right)=\frac{1}{4 \pi} \oint_{C} \oint_{C^{\prime}}\left[\frac{d x(t)}{d t} \frac{d y(s)}{d s}\right] \cdot \frac{x(t)-y(s)}{|x(t)-y(s)|^{3}} d t d s .
$$

Эта интегральная формула была предложена Гауссом без доказательства в работе [5]. Согласно специальным историческим исследованиям [6] Гаусс интересовался числом зацепления земной орбиты с орбитами астероидов. Его аргументация вполне может быть изложена на современном математическом языке (см., например, [3]). Более того, в среде специалистов есть убеждение (см., например, [7]), что Гаусс знал другое, электродинамическое, доказательство этой формулы: пропустить ток через первую петлю и вычислить циркуляцию магнитного поля по второй петле. В силу закона Ампера эта циркуляция равна полному току, ограниченному второй петлей, который равен току, протекающему вдоль первой петли, умноженному на число зацепления этих петель. Примененив формулу Био-Савара для магнитного поля

$$
\mathbf{A}(x)=\frac{1}{4 \pi} \int_{\mathbb{R}^{3}}\left[\mathbf{B}\left(x^{\prime}\right) \frac{x-x^{\prime}}{\left|x-x^{\prime}\right|^{3}}\right] d x^{\prime},
$$

получаем интеграл зацепления Гаусса, а точнее, величину, которая называется магнитной спиральностью. Поэтому вполне можно сказать, что идея рассмотрения спиральности для магнитного поля принадлежит Гауссу. Тем не менее в этой связи еще часто упоминаются Гельмгольц и Кельвин (см. [8]).

В бесконечном пространстве спиральность может быть введена для любого бездивергентного поля с правильными граничными условиями на бесконечности.

В 50-е годы ХХ века вопросы, связанные со спиральностью в идеальной МГд, изучались в работах [9], [10]. Явное выражение спиральности $H=2 n \Phi_{1} \Phi_{2}$ магнитного поля, носитель которого заключен в двух трубках с потоками $\Phi_{1}, \Phi_{2}$, через интеграл зацепления Гаусса $n=\operatorname{lk}\left(C, C^{\prime}\right)$, было получено в работах [11], [12]. Название "магнитная спиральность" для величины $H$ было введено Моффатом [12]. В предположении, что силовые линии магнитного поля В замкнуты, в [12] (см. также [13]) магнитная спиральность была интерпретирована как среднее попарное зацепление силовых линий поля В. Этому вопросу посвящена работа [14], которую следует считать "мостиком" между теорией узлов и магнитной гидродинамикой. 
Целью настоящей работы является изучение геометрического и топологического смысла токовой спиральности

$$
\chi^{c}=\int \mathbf{B} \cdot \operatorname{rot} \mathbf{B} d x
$$

(см. теорему в разделе 4) и ее физического проявления. Эта задача важна в первую очередь потому, что с помощью токовой спиральности формулируется закон изменения магнитной спиральности $\chi^{\mathrm{m}}$ в диссипативной среде: $d \chi^{\mathrm{m}} / d t=-2 \eta \chi^{\mathrm{c}}$. Это соотношение хорошо известно (см., например, [1], [4], [14]). В настоящей работе приводится его подробный вывод в лемме 1 (см. раздел 3$)$.

Наш подход к этой задаче основан на анализе Моффата и Рико [15] формулы Калугариану [16], [17], позволяющей представить магнитную спиральность в виде

$$
\chi^{\mathrm{m}}=(\mathrm{Tw}+\mathrm{Wr}) \Phi^{2} .
$$

В разделе 4 , записав с помощью локальных цилиндрических координат $(r, \lambda, z)$ компоненты магнитного поля в виде

$$
\mathbf{B}(r, \lambda, z)=\left(0, \frac{2 \pi r}{L} \kappa \theta(r), \theta(r)\right),
$$

мы интерпретируем величину Тw скручивания магнитной трубки длины $L$ с помощью коэффициента скручивания $\kappa$ :

$$
\mathrm{Tw}=\kappa .
$$

Полученная в настоящей работе приближенная формула $\chi^{\mathrm{c}} \approx 2 \rho \mathrm{Tw} \Phi^{2}$ (ср. формулу (10) в разделе 4) показывает, что спиральность силовых линий поля плотности тока $\mathbf{j}$ пропорциональна скручиванию Тw силовых линий магнитного поля В. На этой основе удалось выяснить геометрическую причину изменения магнитной спиральности в среде с ненулевым коэффициентом магнитной проницаемости $\eta$. Известно, что в диссипативной среде магнитная спиральность убывает (см. приведенную выше формулу для $\left.d \chi^{\mathrm{m}} / d t\right)$. Однако теперь мы можем сказать, что магнитная спиральность убывает тем быстрее, чем более скручены силовые линии магнитного поля В в трубках (см. далее формулу (12) в разделе 4). При этом величина Wr не влияет на скорость диссипации магнитной спиральности.

В разделе 5 мы рассматриваем обобщение магнитной гидродинамики, предложенное в [18]. Речь идет о модификации уравнения Фарадея с целью учета слабых взаимодействий. В этом случае для эволюции спиральности справедлива формула (14). По нашему мнению, другие важные следствия, вытекающие из работы [18], могут быть дополнительно найдены в последующих исследованиях.

\section{2. ПРЕДВАРИТЕЛЬНОЕ ОПИСАНИЕ МАГНИТНЫХ ТРУБОК}

Пусть $V=D^{2}\left(r_{0}\right) \times \mathbb{R}^{1} \subset \mathbb{R}^{3}$ - бесконечный открытый цилиндр в евклидовом пространстве $\mathbb{R}^{3}$, снабженный цилиндрической системой координат $(r, \lambda, z)$, где $0 \leqslant r<r_{0}$. Рассмотрим сдвиг $t: V \rightarrow V$, определенный в выбранной системе координат формулой $t(r, \lambda, z)=\left(r, \lambda+\lambda_{0}, z+z_{0}\right)$ при некоторых фиксированных значениях 
констант $\lambda_{0}, z_{0}$ (удобно считать, что $z_{0}=2 \pi$ ). В этом случае определен полноторий $U=V / t$, который служит базой циклического накрытия $V \rightarrow U$. Многообразие $U$ наделяется римановой метрикой, индуцированной стандартной метрикой цилиндра $V$. Индуцированные координаты на $U$ обозначим так же, как и координаты на $V$ с учетом неоднозначности при факторизации.

Определим на $V$ инвариантное при трансляции вдоль вертикальной оси $z$ векторное поле $\mathbf{B}^{V}: V \rightarrow \mathbb{R}^{3}$, полагая $\mathbf{B}^{V}(r, \lambda, z)=(0,0, \theta(r))$. При этом считаем, что задана функция интенсивности поля $\mathbf{B}^{V}$ - гладкая невозрастающая функция $\theta$ : $[0,+\infty) \rightarrow\left[0, \theta_{0}\right]$, где $\theta_{0}>0, \theta_{0}=\theta(0) \geqslant \theta(r) \geqslant \theta\left(r_{0}\right)=0, r \in[0,+\infty)$ (см. рисунок).

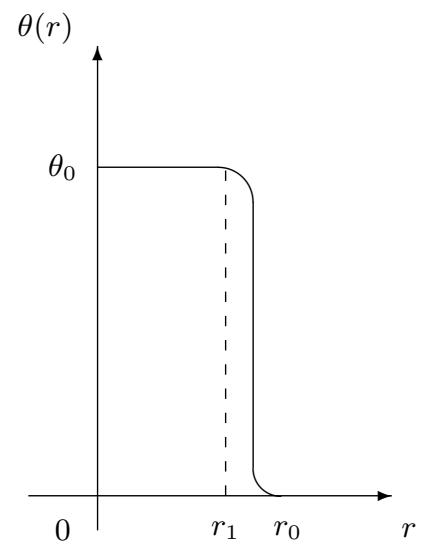

Очевидно, что $\operatorname{div}\left(\mathbf{B}^{V}\right)=0, \operatorname{rot}\left(\mathbf{B}^{V}\right)=\left(0,-\theta^{\prime}(r), 0\right)$. Поскольку поле $\mathbf{B}^{V}$ инвариантно при сдвиге $t$, при проекции $V \rightarrow U$ поле $\mathbf{B}^{V}$ факторизуется до бездивергентного (в другой терминологии, соленоидального) векторного поля $\mathbf{B}$ на $U$. Поля $\mathbf{B}$ и $\operatorname{rot}\left(\mathbf{B}^{V}\right)$ являются касательными к краю трубки $U$. Кроме того, очевидно, они касательны к краю каждой из вложенных коаксиальных трубок $U(r)=V(r) / t$ радиуса $r, 0<r<r_{0}, V(r)=D^{2}(r) \times \mathbb{R}^{1} \subset V$.

Образ произвольного гладкого вложения $\iota: U \hookrightarrow \mathbb{R}^{3}$, сохраняющего объем, будем называть магнитной трубкой. По определению она представляет собой (возможно, заузленный) полноторий (далее также обозначаемый через $U$ ), снабженный векторным полем $\iota_{*}(\mathbf{B})$ (далее его будем обозначать $\mathbf{B}$ ), касающимся края магнитной трубки. Предположим, что наибольший радиус поперечного сечения трубки $U$ много меньше наименьшего радиуса кривизны ее центральной линии (в дальнейшем такую трубку будем называть тонкой).

При этом потоком (или интенсивностью) бездивергентного векторного поля В в трубке $U$ назовем число $\Phi$, равное потоку векторного поля $\mathbf{B}$ через любое трансверсальное сечение трубки $U$. В нашем случае получаем

$$
\Phi=2 \pi \int_{0}^{r_{0}} r \theta(r) d r .
$$

При условии $\operatorname{div} \mathbf{B}=0$ определен вектор-потенциал $\mathbf{A}$ поля $\mathbf{B}: \operatorname{rot} \mathbf{A}=\mathbf{B}$. Потребуем, чтобы

$$
\mathbf{A}(r) \sim r^{-2} \quad \text { при } \quad r \rightarrow \infty .
$$


В общем случае, как известно, вектор-потенциал определен с точностью до калибровочного преобразования $\mathbf{A} \rightarrow \mathbf{A}+\operatorname{grad} f$, где $f: \mathbb{R}^{3} \rightarrow \mathbb{R}-$ произвольная гладкая функция. Кулоновская калибровка $\operatorname{div} \mathbf{A}=0$ и граничные условия (1) определяют (нелокальное) векторное поле $\mathbf{A}$ однозначно.

Вектор-потенциал $\mathbf{A}_{0}^{V}$ поля $\mathbf{B}^{V}$ внутри бесконечной прямой трубки $V$ можно определить стандартным способом по формуле

$$
\mathbf{A}_{0}^{V}=\left(0, A_{\lambda}(r), 0\right), \quad A_{\lambda}\left(r^{\prime}\right)=\frac{1}{r^{\prime}} \int_{0}^{r^{\prime}} r \theta(r) d r .
$$

Отметим, что $\mathbf{A}_{0}^{V}$ на боковой поверхности бесконечной трубки $V$ не обращается в нуль, но на центральной линии трубки $\mathbf{A}_{0}^{V}$ обращается в нуль и не имеет особенности в окрестности этой центральной линии.

Легко получить явную формулу вектор-потенциала А поля В во всем пространстве $\mathbb{R}^{3}$. Для вектор-потенциала $\mathbf{A}$ при ограничении на $U$ имеем разложение

$$
\mathbf{A}=\mathbf{A}_{0}+\operatorname{grad} \phi_{0}+\operatorname{grad} \phi,
$$

где $\phi_{0}$ - линейная функция, которая зависит лишь от координаты $z, \phi$ - произвольная гладкая $t$-периодическая функция с нулевым средним значением.

Векторное поле $\mathbf{A}_{0}^{V}$ на $V$ инвариантно относительно сдвига $t$ и факторизуется до поля $\mathbf{A}_{0}$ в трубке $U$, для которого $\operatorname{rot}\left(\mathbf{A}_{0}\right)=\mathbf{B}$. Поэтому предыдущую формулу можно также применить для замкнутой магнитной трубки. При этом потенциал $\phi_{0}$ становится линейной многозначной функцией, а однозначная функция $\phi$ удовлетворяет уравнению

$$
\oint \operatorname{grad} \phi \cdot d \mathbf{l}=0
$$

при интегрировании по центральной линии $\mathbf{l}=\mathbf{l}(\nu)$ трубки $U, \nu=z\left(\bmod z_{0}\right)$, $0 \leqslant \nu \leqslant z_{0}$

\section{3. ОБ УРАВНЕНИЯХ И ИНТЕГРАЛАХ МАГНИТОГИДРОДИНАМИКИ}

В МГД-приближении электромагнитное поле в проводящей жидкой движущейся среде определяется системой уравнений [19]:

$$
\begin{aligned}
\operatorname{rot} \mathbf{E} & =-\frac{1}{c} \frac{\partial \mathbf{B}}{\partial t}, \\
\operatorname{rot} \mathbf{H} & =\frac{4 \pi}{c} \mathbf{j}, \\
\operatorname{div} \mathbf{B} & =0, \\
\mathbf{j} & =\sigma\left(\mathbf{E}+\frac{[\mathbf{v}, \mathbf{B}]}{c}\right),
\end{aligned}
$$

где $\sigma$ - проводимость среды, $\mathbf{E}$ - электрическое поле, $\mathbf{H}$ и $\mathbf{B}$ - соответственно напряженность магнитного поля и его магнитная индукция, $\mathbf{j}$ - плотность тока, $\mathbf{v}(\mathbf{r}, t)-$ скорость движения среды. Уравнение (2) выражает закон Фарадея, уравнение (3) закон Био-Савара, а уравнение (5) - закон Ома. 
Рассмотрим однородную среду с постоянными проводимостью $\sigma$ и магнитной проницаемостью $\mu \equiv 1$. В этом случае $\mathbf{H}=\mathbf{B}$ и с учетом уравнений (2), (5) и (3) получаем уравнение индукции

$$
\frac{\partial \mathbf{B}}{\partial t}=\operatorname{rot}[\mathbf{v}, \mathbf{B}]-\eta \operatorname{rot} \operatorname{rot} \mathbf{B},
$$

где $\eta=c^{2} /(4 \pi \sigma)-$ коэффициент магнитной диффузии.

В случае $\eta=0$ среда называется идеальной, а из уравнения (6) следует "вмороженность" магнитного поля В, т.е. в идеальном случае уравнение (6) эквивалентно уравнению $\left(\partial / \partial t+\mathcal{L}_{\mathbf{v}}\right) B^{(2)}=0$ для 2 -формы $B^{(2)}=i_{\mathbf{B}} \mu$, ассоциированной с векторным полем $\mathbf{B}$ при помощи выбранной формы объема $\mu$. Для краткости записи положим далее $c=1$.

Определим калибровочно инвариантные псевдоскалярные величины: интеграл магнитной спиральности

$$
\chi^{\mathrm{m}}=\int_{U} \mathbf{A} \cdot \mathbf{B} d x
$$

и интеграл токовой спиральности

$$
\chi^{\mathrm{c}}=\int_{U} \mathbf{B} \cdot \operatorname{rot} \mathbf{B} d x
$$

Последний интеграл можно также записать в силу (3) как

$$
\chi^{\mathrm{c}}=4 \pi \int_{U} \mathbf{B} \cdot \mathbf{j} d x .
$$

Лемма 1. Справедливо тождество

$$
\frac{d \chi^{\mathrm{m}}}{d t}=-2 \eta \chi^{\mathrm{c}}
$$

В частности, в предположении $\eta=0$ интеграл $\chi^{\mathrm{m}}$ сохраняется.

ДокАЗАтЕЛЬство. Закон сохранения $\chi^{\mathrm{m}}$ в идеальном случае вытекает из формулы(7) при $\eta=0$. Докажем эту формулу. Переходя к потенциалам в формуле (2), получаем

$$
\frac{\partial \mathbf{A}}{\partial t}=-\mathbf{E}-\operatorname{grad} \varphi
$$

Следовательно,

$$
\begin{aligned}
\frac{\partial}{\partial t}(\mathbf{A} \cdot \mathbf{B}) & =(-\mathbf{E}-\operatorname{grad} \varphi) \cdot \mathbf{B}-\mathbf{A} \cdot \operatorname{rot} \mathbf{E}= \\
& =-\mathbf{E} \cdot \mathbf{B}-\operatorname{div}(\varphi \mathbf{B})-\operatorname{div}[\mathbf{E}, \mathbf{A}]-\mathbf{E} \cdot \mathbf{B}=-2 \mathbf{E} \cdot \mathbf{B}+\operatorname{div}(-\varphi \mathbf{B}-[\mathbf{E}, \mathbf{A}])
\end{aligned}
$$

Таким образом,

$$
\frac{d \chi^{\mathrm{m}}}{d t}=\frac{d}{d t} \int_{U} \mathbf{A} \cdot \mathbf{B} d x=-2 \int_{U} \mathbf{E} \cdot \mathbf{B} d x .
$$

Учитывая уравнения (5) и (3), находим $\mathbf{E}$ по формуле

$$
\mathbf{E}=-[\mathbf{v}, \mathbf{B}]+\eta \operatorname{rot} \mathbf{B} .
$$


Подставив это выражение в уравнение (8), получаем

$$
\frac{d \chi^{\mathrm{m}}}{d t}=2 \int_{U}([\mathbf{v}, \mathbf{B}]-\eta \operatorname{rot} \mathbf{B}) \cdot \mathbf{B} d x=-2 \eta \int_{U} \mathbf{B} \cdot \operatorname{rot} \mathbf{B} d x=-2 \eta \chi^{\mathrm{c}}
$$

ЗАмЕчАниЕ. Выражение для скорости диссипации магнитной спиральности, указанное в лемме 1, хорошо известно (см., например, [1], [4], [14]). Доказательство здесь приводится для удобства читателя. Кроме того, оно потребуется нам при обсуждении в разделе 5 формулы (14) - аналога формулы (7) для крупномасштабного магнитного поля ранней Вселенной, описывающей скорость роста спиральности при учете слабых взаимодействий.

\section{4. ЭВОЛЮЦИЯ МАГНИТНЫХ ТРУБОК ПРИ НАЛИЧИИ МАГНИТНОЙ ДИФФУЗИИ}

Нашей целью в этом разделе является выяснение топологического и физического смысла интеграла токовой спиральности $\chi^{\mathrm{c}}$.

Напомним, что величина скручивания трубки Tw вычисляется по формуле

$$
\mathrm{Tw}=\frac{1}{2 \pi} \oint_{C}\left[\mathbf{u}(s), \frac{d \mathbf{u}(s)}{d s}\right] \cdot \mathbf{t}(s) d s,
$$

где $\mathbf{t}(s)$ - касательный вектор единичной длины к $C=l$ - центральной линии магнитной трубки $U$ с натуральной параметризацией $\mathbf{a}=\mathbf{a}(s) ; \mathbf{u}(s)$ - единичный вектор нормали к центральной линии, восстановленный в направлении другой силовой линии $C^{\prime}$ магнитного поля, располагающейся на коаксиальной магнитной торической поверхности радиуса $r, 0<r<r_{0}$, в трубке $U$ (т.е. $\mathbf{u}(s)$ - образующая рассматриваемой линейчатой поверхности с направляющей $\mathbf{a}(s))$; силовая линия $C^{\prime}$ записывается в виде $\mathbf{a}(s)+r \mathbf{u}(s)$.

Напомним также, что величина перекручивания трубки $\mathrm{Wr}$ определяется только поведением центральной линии $l$ и задается интегралом:

$$
\mathrm{Wr}=\frac{1}{4 \pi} \oint_{C} \oint_{C}\left[\frac{d \mathbf{a}(s)}{d s}, \frac{d \mathbf{a}\left(s^{\prime}\right)}{d s^{\prime}}\right] \cdot \frac{\mathbf{a}(s)-\mathbf{a}\left(s^{\prime}\right)}{\left|\mathbf{a}(s)-\mathbf{a}\left(s^{\prime}\right)\right|^{3}} d s d s^{\prime} .
$$

Величина Wr была введена Калугариану [16], [17], а Tw была введена Фуллером [20].

Величины $\mathrm{Tw}$ и $\mathrm{Wr}$ в отличие от коэффициента зацепления не являются топологически инвариантными, но, как следует из интегральных выражений, при непрерывной деформации трубки они меняются непрерывно.

Спиральность $\chi^{\mathrm{m}}$ поля В в замкнутой трубке $U$ можно представить в виде (см. [4], [14], [15], [21])

$$
\chi^{\mathrm{m}}=\operatorname{lk}\left(C, C^{\prime}\right) \Phi^{2}=(\mathrm{Tw}+\mathrm{Wr}) \Phi^{2} .
$$

Можно сказать, что в МГД формула (9) для магнитной спиральности $\chi^{\mathrm{m}}$ является аналогом геометрической формулы Калугариану [16], [17] и, более того, ее также принято называть формулой Калугариану. 
Докажем следующее утверждение.

ЛЕмма 2. Для трубки со средним радиусом $r_{0}$ нормального сечения справедлива оченка

$$
\chi^{\mathrm{c}} \approx \frac{4}{r_{0}^{2}} \mathrm{Tw} \Phi^{2} .
$$

ДоказАтельство. Рассмотрим сначала случай магнитного поля, имеющего $(r, \lambda, z)$-компоненты

$$
\mathbf{B}^{V}=\left(0, \frac{2 \pi r}{L} \kappa \theta(r), \theta(r)\right)
$$

в бесконечно длинной прямой трубке $V$ радиуса $r_{0}$. В уравнении $(11)$ константа $L$ в дальнейшем будет соответствовать длине трубки, $\theta(r)$ - функция интенсивности поля $\mathbf{B}^{V}$ внутри трубки (график которой показан на рисунке), $\kappa>0$ - коэффициент скручивания трубки вокруг центральной оси. В цилиндрической системе координат $(r, \lambda, z)$ трубка $V$ может быть задана следующими условиями: $0 \leqslant r<r_{0}$, $0 \leqslant \lambda \leqslant 2 \pi,-\infty<z<+\infty$.

Из (11) следует вид компонент поля $\operatorname{rot} \mathbf{B}^{V}$ :

$$
\begin{gathered}
\left(\operatorname{rot} \mathbf{B}^{V}\right)_{r}=0, \quad\left(\operatorname{rot} \mathbf{B}^{V}\right)_{\lambda}=-\theta^{\prime}(r), \\
\left(\operatorname{rot} \mathbf{B}^{V}\right)_{z}=4 \frac{\pi \kappa}{L} \theta(r)+r \frac{2 \pi \kappa}{L} \theta^{\prime}(r) .
\end{gathered}
$$

Обозначив через $S$ трансверсальное сечение трубки, заданное по формуле $z=z_{0}$, получаем

$$
\int_{S} \mathbf{B}^{V} \cdot \operatorname{rot} \mathbf{B}^{V} r d r d \lambda \approx 4 \pi^{2} \frac{\kappa}{L} \theta_{0}^{2} r_{0}^{2} .
$$

Учитывая для потока вектора В через трансверсальное сечение $S$ трубки $U$ приближенную оценку $\Phi \approx \pi r_{0}^{2} \theta_{0}$, получаем приближенное равенство

$$
\int_{S} \mathbf{B}^{V} \cdot \operatorname{rot} \mathbf{B}^{V} r d r d \lambda \approx \frac{4}{L r_{0}^{2}} \kappa \Phi^{2} .
$$

Осталось заметить, что на отрезке трубки длины $L$ значение $\kappa$ совпадает с интегралом Tw, что доказывает формулу (10) для прямой магнитной трубки.

Рассмотрим теперь общий случай трубки, полученной как образ $\iota(U) \subset \mathbb{R}^{3}$ стандартной трубки при помощи вложения $\iota$, сохраняющего объем. Обозначим через $L$ длину центральной линии и предположим, что трубка достаточно тонкая (по отношению к кривизне центральной линии).

В соответствии с результатами работы [15] поле $\mathbf{B}$ в трубке $U$ разлагается в сумму $\mathbf{B}=\mathbf{B}_{\mathrm{a}}+\mathbf{B}_{\mathrm{m}}$, где $\mathbf{B}_{\mathrm{a}}-$ поле, параллельное касательному вектору к центральной линии трубки, $\mathbf{B}_{\mathrm{m}}$ - поле в плоскости нормального сечения, перпендикулярное такому касательному вектору. Удобно ввести векторное поле $\xi$ вдоль центральной линии, перпендикулярное этой линии, такое, что его производная по направлению касательного вектора к центральной линии равна нулю.

Разрежем трубку на бесконечно большое число $\Delta$ цилиндрических участков длины $L_{k}=L / \Delta, k=1,2, \ldots, \Delta$. В каждом бесконечно тонком цилиндре введем цилиндрическую систему координат, связанную с полем $\xi$ и проделаем предыдущие 
вычисления. Поскольку поправка на кривизну и кручение трубки имеют более высокий порядок малости, чем точность вычисления, бесконечно тонкую трубку можно считать плоской. Оценка (10) доказана.

Итак, можно оценить полученный результат с точки зрения МГД следующим образом: формула (10) для токовой спиральности дополняет формулу (7) для магнитной спиральности. Мы видим, в частности, что токовая спиральность не зависит от значения величины Wr и равна нулю при нулевом значении скрученности $\mathrm{Tw}$ магнитных силовых линий.

Теперь, используя формулу (7), получаем

СледСтвиЕ. Имеет место следующее приближенное равенство:

$$
\frac{d \chi^{\mathrm{m}}}{d t} \approx-\eta \rho \mathrm{Tw} \Phi^{2}
$$

где $\rho=2 / r_{0}^{2}$.

С физической точки зрения равенство (12) означает, что в присутствии магнитной диффузии $(\eta \neq 0)$ магнитные трубки раскручиваются относительно центральной оси (магнитная спиральность $\chi^{\mathrm{m}}$ убывает), причем скорость раскручивания магнитной трубки, определяемая значением правой части равенства (12), пропорциональна среднему значению скручивания, которое определяется левой частью равенства. Перемещение трубки без раскручивания происходит в идеальном случае (т.е. при $\eta=0)$, при этом разложение в сумму $\chi^{\mathrm{m}}=(\mathrm{Wr}+\mathrm{Tw}) \Phi^{2}$ для трубки сохраняется. Как следует из формулы (12), при ненулевом значении магнитной диффузии $\eta$ магнитная трубка раскручивается за счет изменения первого слагаемого Tw, которое не компенсируется изменением второго слагаемого Wr. Скорость раскручивания трубки прямо пропорциональна коэффициенту магнитной диффузии и значению интеграла $\mathrm{Tw}$, который является мерой скручивания магнитной трубки.

\section{5. МАГНИТНОЕ ПОЛЕ РАННЕЙ ВСЕЛЕННОЙ}

Этот раздел посвящен приложению наших оценок к геометрическому объяснению роста напряженности крупномасштабного магнитного поля в ранней Вселенной (на лептонной стадии).

Напомним, что в недавней работе Семикоза и Соколова [18] в рамках Стандартной модели с нейтрино и антинейтрино предложен бесстолкновительный механизм продуцирования магнитной спиральности через коллективное взаимодействие нейтрино и горячей электрон-позитронной плазмы. В такой модели имеет место обобщенное уравнение индукции (см., например, [22]), описывающее эволюцию среднего магнитного поля с $\alpha$-эффектом,

$$
\frac{\partial \mathbf{B}}{\partial t}=\operatorname{rot}[\mathbf{v}, \mathbf{B}]+\alpha \operatorname{rot} \mathbf{B}-\eta \operatorname{rot} \operatorname{rot} \mathbf{B} .
$$

Это уравнение получается, как и уравнение (6), из (2) и (3), но в выражении для напряженности электрического поля $\mathbf{E}$ кроме слагаемых, входящих в уравнение (5), дополнительно учтен вклад слабых взаимодействий $\mathbf{E}_{\mathrm{weak}}=-\alpha \mathbf{B}$ (см. [23]), т.е.

$$
\mathbf{E}=4 \pi \eta \mathbf{j}-[\mathbf{v}, \mathbf{B}]-\alpha \mathbf{B} .
$$


В этом варианте обобщения стандартной МГД для скорости роста магнитной спиральности в [18] получен следующий аналог формулы (7):

$$
\frac{d}{d t} \chi^{\mathrm{m}}=-2 \eta \chi^{\mathrm{c}}+2 \alpha \int_{U} \mathbf{B}^{2} d x .
$$

Поясним, что при учете слабых взаимодействий, предложенном в выражении (13), формулу (14) можно вывести тем же стандартным способом, что и формулу (7) (а именно, изложенным в доказательстве леммы 1). При этом масштаб рассматриваемого поля сравним с горизонтом (но меньше его), и магнитные силовые линии замкнуты в области интегрирования $U$.

Основные результаты работы [18] сводятся к следующему. В горячей плазме ранней Вселенной, когда коэффициент $\alpha$ достаточно велик, магнитная спиральность принимает существенные значения (см. оценку (9) в [18]). Этот факт может объяснить формирование (прото)галактических структур в однородной и изотропной Вселенной на лептонной стадии. При остывании плазмы величина $\alpha \rightarrow 0$, продуцирование магнитной спиральности становится невозможным и фаза первичного формирования галактик и скоплений галактик должна перейти в следующую фазу их развития.

Формулу (14) можно дополнить следующей:

$$
\frac{d}{d t} \int_{U} \mathbf{B}^{2} d x=2 \int_{U} \mathbf{B} \cdot \operatorname{rot}[\mathbf{v}, \mathbf{B}] d x-2 \eta \int_{U}(\operatorname{rot} \mathbf{B})^{2} d x+2 \alpha \chi^{\mathrm{c}} .
$$

В справедливости (15) легко убедиться непосредственно:

$$
\begin{aligned}
\frac{d}{d t} \int \mathbf{B}^{2} d x & =2 \int \mathbf{B} \cdot \frac{\partial \mathbf{B}}{\partial t} d x=2 \int \mathbf{B} \cdot(-\operatorname{rot} \mathbf{E}) d x= \\
& =2 \int \mathbf{B} \cdot(-\eta \operatorname{rot} \operatorname{rot} \mathbf{B}+\operatorname{rot}[\mathbf{v}, \mathbf{B}]+\alpha \operatorname{rot} \mathbf{B}) d x= \\
& =-2 \eta \int \mathbf{B} \cdot(\operatorname{rot} \operatorname{rot} \mathbf{B}) d x+2 \int \mathbf{B} \cdot \operatorname{rot}[\mathbf{v}, \mathbf{B}] d x+2 \alpha \int \mathbf{B} \cdot \operatorname{rot} \mathbf{B} d x= \\
& =2 \int \mathbf{B} \cdot \operatorname{rot}[\mathbf{v}, \mathbf{B}] d x-2 \eta \int(\operatorname{rot} \mathbf{B})^{2} d x+2 \alpha \chi^{\mathrm{c}} .
\end{aligned}
$$

Заметим теперь, что в предположении $\alpha \gg \eta$ (выполняющемся на рассматриваемой стадии развития Вселенной) второе слагаемое в формуле (15) становится пренебрежимо малым по сравнению с третьим. Третье слагаемое показывает, что энергия

$$
\frac{1}{2} \int \mathbf{B}^{2} d x
$$

магнитного поля В увеличивается в правозакрученных трубках пропорционально скручиванию магнитной трубки (в силу формулы (10)). При наличии достаточного числа правозакрученных трубок наблюдается рост космологического магнитного поля. Очевидно, это увеличивает второе слагаемое правой части формулы (15) и является дополнительным фактором увеличения магнитной спиральности в рассматриваемый период. 
Благодарности. Авторы благодарят И.С. Красильщика, Д.Д. Соколова и В. Б. Семикоза за обсуждения. Работа П. М. Ахметьева частично поддержана РФФИ (грант № 08-01-00663) и фондом INTAS (грант № 05-1000008-7805).

\section{Список литературы}

[1] H. K. Moffatt, Magnetic Field Generation in Electrically Conducting Fluids, Cambridge Univ. Press, Cambridge, 1978.

[2] Э. Прист, Т. Форбс, Магнитное пересоединение. Магнитогидродинамическая теория и приложения, Физматлит, М., 2005.

[3] В. И. Арнольд, "Асимптотический инвариант Хопфа и его приложения", Материаль Всесоюзной школы по дифференииальным уравнениям с бесконечным числом независимых переменных и по динамическим системам с бесконечным числом степеней свободы (Дилижан, 21 мая - 3 июня 1973 г.), АН Арм. ССР, Ереван, 1974, 229-256; Избранное-60, Фазис, М., 1997, 215-236.

[4] В.И. Арнольд, Б.А. Хесин, Топологические методы в магнитной гидродинамике, МЦНМО, М., 2007.

[5] C. F. Gauss, Werke. B. V, Springer, Göttingen, 1867.

[6] M. Epple, Math. Intellingencer, 20:1 (1998), 45-52.

[7] D. DeTurck, H. Gluck, The Gauss linking integral on the 3-sphere and in hyperbolic 3-space, arXiv: math/0406276v1.

[8] W. Thomson, Trans. R. Soc. Edin., XXV (1869), 217-260; W. Thomson, "Maximum and minimum energy in vortex motion", Mathematical and Physical Papers. V. IV. Hydrodynamics and General Dynamics, Cambridge Univ. Press, Cambridge, 1910, 172-183.

[9] L. Woltjer, Proc. Natl. Acad. Sci. USA, 44:6 (1958), 489-491.

[10] W. M. Elsasser, Rev. Modern Phys., 28:2 (1956), 135-163.

[11] J. J. Moreau, C. R. Acad. Sci. Paris, 252 (1961), 2810-2812.

[12] H. K. Moffatt, J. Fluid Mech., 35 (1969), 117-129.

[13] H. K. Moffatt, J. Fluid Mech., 106 (1981), 27-47.

[14] M. A. Berger, G. B. Field, J. Fluid Mech., 147 (1984), 133-148.

[15] H. K. Moffatt, R. L. Ricca, Proc. R. Soc. Lond. Ser. A, 439:1906 (1992), 411-429.

[16] G. Călugărianu, Rev. Math. Pures Appl., 4 (1959), 5-20.

[17] G. Călugărianu, Czechoslovak Math. J., 11(86) (1961), 588-625.

[18] V. B. Semikoz, D. D. Sokoloff, Astron. Astrophys., 433:3 (2005), L53-L56.

[19] Л. Д. Ландау, Е. М. Лифшиц, Теоретическая физика. Т. 8. Электродинамика сплошных сред, Наука, М., 1982.

[20] F. B. Fuller, Proc. Natl. Acad. Sci. USA, 68:4 (1971), 815-819.

[21] M. R. Dennis, J. H. Hannay, Proc. R. Soc. Lond. Ser. A, 461:2062 (2005), 3245-3254.

[22] А. А. Рузмайкин, Д. Д. Соколов, А. М. Шукуров, Магнитные поля галактик, Наука, M., 1988.

[23] V.B. Semikoz, Lepton MHD and magnetic field generation in SM, arXiv: hep-ph/0403096.

Поступила в редакцию 10.10.2007, после доработки 8.05.2008 